\title{
Dipole and quadrupole solitons in optically-induced two-dimensional defocusing photonic lattices
}

\author{
H. Susanto ${ }^{\text {a }}$, K. Law ${ }^{\text {a }}$, P.G. Kevrekidis ${ }^{\text {a, }}$, L. Tang ${ }^{\text {b }}$, C. Lou ${ }^{\text {, }}$, \\ X. Wang ${ }^{b, c}$ Z. Chen ${ }^{b, c}$ \\ ${ }^{a}$ Department of Mathematics and Statistics, University of Massachusetts, \\ Amherst, MA 01003, USA \\ ${ }^{\mathrm{b}}$ Key Laboratory for Weak-Light Nonlinear Photonics Materials, Ministry of \\ Education, and TEDA Applied Physical School, Nankai University, Tianjin \\ 300457, China \\ ${ }^{\mathrm{c}}$ Department of Physics and Astronomy, San Francisco State University, \\ San Francisco, CA 94132, USA
}

\begin{abstract}
Dipole and quadrupole solitons in a two-dimensional optically induced defocusing photonic lattice are theoretically predicted and experimentally observed. It is shown that in-phase nearest-neighbor and out-of-phase next-nearest-neighbor dipoles exist and can be stable in the intermediate intensity regime. There are also different types of dipoles that are always unstable. In-phase nearest-neighbor quadrupoles are also numerically obtained, and may also be linearly stable. Out-of-phase, nearestneighbor quadrupoles are found to be typically unstable. These numerical results are found to be aligned with the main predictions obtained analytically in the discrete nonlinear Schrödinger model. Finally, experimental results are presented for both dipole and quadrupole structures, indicating that self-trapping of such structures in the defocusing lattice can be realized for the length of the nonlinear crystal (10 $\mathrm{mm})$.
\end{abstract}

Key words: dipoles, quadrupoles, solitons, defocusing photonic lattices PACS: 00.00.-o

\footnotetext{
* Corresponding author.

Email address: kevrekid@math.umass.edu (P.G. Kevrekidis).
} 


\section{Introduction}

Self-trapping of light in photonic lattices optically induced in nonlinear photorefractive crystals, such as strontium barium niobate (SBN) has attracted a considerable amount of attention, ever since its theoretical inception [1] and experimental realization $[2,3,4]$. This is perhaps primarily because it constitutes a setting where it is very natural to consider the competing effects of nonlinearity with diffraction and to study the effects of periodic "potentials" on solitary waves; here the role of the "potential" is played by the ordinary polarization of light forming a waveguide array in which the extra-ordinarily polarized probe beam evolves.

This setting has provided a fertile ground for the experimental realization and detailed examination of many interesting concepts of nonlinear wave physics, including, for instance, the formation of discrete dipole [5], necklace [6] solitons and even stripe patterns [7], rotary solitons [8], discrete vortices [9] or the realization of photonic quasicrystals [10] and Anderson localization [11]. It is clear from these findings that this setting can serve not only as a host for the examination of localized structures that may be usable as carriers and conduits for data transmission and processing in all-optical communication schemes; it is also relevant as an experimentally tunable playground where numerous fundamental issues of solitons and nonlinear waves can be explored.

It is interesting to mention in passing that in parallel (and nearly concurrently) to this direction of photorefractive crystal lattices, a number of other venues were developed in optical and atomic physics, where the interplay of nonlinearity with periodicity is important for the observed dynamics. Such contexts involve on the optical end, the numerous developments on the experimental and theoretical investigation of optical waveguide arrays; see e.g. $[12,13]$ for relevant reviews. On the atomic physics end, the confinement of dilute alkali vapors in optical lattice potentials [14] has similarly offered the opportunity to examine many fundamental phenomena involving spatial periodicity, including the manifestation of modulational instabilities, Bloch oscillations, Landau-Zener tunneling and gap solitons among others; see [15] for a recent review.

In the present study, motivated by SBN crystals and photorefractive lattices, we focus on two-dimensional periodic, nonlinear media. In such settings, the vast majority of studies has centered around focusing nonlinearities; the reason for this is two-fold: on the theoretical end, the topic of focusing solitary waves is interesting due to the collapse (and concomitant arrest by the lattice) phenomena in the case of cubic nonlinearity [16]. On the other hand, in the photorefractive case, collapse is absent due to the saturable nature of the nonlinearity; however, in the latter case, it is technically easier to work 
with voltages that are in the regime of focusing rather than in that of the defocusing nonlinearity (in the latter case, sufficiently large voltage, which is tantamount to large nonlinearity, may cause damage to the crystal). Hence, the only coherent structure that appears to be explored in the defocusing regime experimentally appears to be that of fundamental gap solitons excited in the vicinity of the edge of the first Brillouin zone [2]. More complex gap structures appear not to have been studied systematically, perhaps partially due to the above reasons. In an earlier work [17], motivated by the above lack of results, we considered more complicated multipole (i.e., dipole and quadrupol 1 ) and vortex structures in the defocusing case for a standard discrete dynamical lattice (namely, the discrete nonlinear Schrödinger equation with cubic nonlinearity [18]). This provided us with an analytically tractable and numerically accessible roadmap for the study of some of the relevant solutions. In the present work, we employ a continuum model involving a periodic potential and a saturable nonlinearity as associated with the SBN crystals. In particular, we demonstrate both experimentally and numerically multipole (dipoles and quadrupoles) solitons in 2D square lattices induced with a selfdefocusing nonlinearity. We numerically analyze both the existence and the stability of these structures and follow their dynamics, in the cases where we find them to be unstable. We also qualitatively compare our findings with the roadmap provided by the discrete model [17].

Our presentation is structured as follows. In section 2, we present our theoretical setup (related to our experiments). Dipole solutions with the two excited sites in adjacent wells of the periodic potential (nearest-neighbor dipoles) are studied in section 3. Subsequently, we do the same for next-nearest-neighbor dipoles (excited in two diagonal sites of the square lattice) in section 4 . Section 5 addresses the case of quadrupoles, and section 6 presents our experimental results for the structures examined. Finally, in section 7 , we summarize our findings, posing some interesting questions for future study.

\section{Setup}

For our theoretical considerations, we use the non-dimensionalized version of the photorefractive model with the saturable nonlinearity, as developed in

\footnotetext{
1 It should be pointed out here that although in the standard electromagnetic convention, one thinks of dipoles as bearing two opposite charges, and quadrupoles as bearing two positive and two negative charges (in our case, phases of the excited sites), here we will use a different notational convention. More specifically, following the etymology of the words, we will refer to any two-site excitation as a dipole, and to any four-site excitation as a quadrupole herein.
} 
detail in $[19,20]$, in the following form:

$$
i U_{z}=-\left(U_{x x}+U_{y y}\right)-\frac{E_{0}}{1+I_{o l}+|U|^{2}} U .
$$

In the above expression $U$ is the slowly varying amplitude of the probe beam normalized by the dark irradiance of the crystal $I_{d}$, and

$$
I_{o l}=I_{0} \cos ^{2}\left(\frac{x+y}{\sqrt{2}}\right) \cos ^{2}\left(\frac{x-y}{\sqrt{2}}\right),
$$

is a square optical lattice intensity function in units of $I_{d}$. Here $I_{0}$ is the lattice peak intensity, $z$ is the propagation distance (in units of $2 k_{1} D^{2} / \pi^{2}$ ), $(x, y)$ are transverse distances (in units of $D / \pi), E_{0}$ is the applied DC field voltage (in units of $\left.\pi^{2}\left(k_{0}^{2} n_{e}^{4} D^{2} r_{33}\right)^{-1}\right), D$ is the lattice spacing, $k_{0}=2 \pi / \lambda_{0}$ is the wavenumber of the laser in the vacuum, $\lambda_{0}$ is the wavelength, $n_{e}$ is the refractive index along the extraordinary axis, $k_{1}=k_{0} n_{e}$, and $r_{33}$ is the electro-optic coefficient for the extraordinary polarization. In line with our experiment, we choose the lattice intensity $I_{0}=5$ (in units of $I_{d}$ ). A plot of the optical lattice is shown in Fig. 1, also for illustrative purposes regarding the location where our localized pulses will be "inserted". In addition, we choose other physical parameters consistently with the experiment as

$$
D=25 \mu \mathrm{m}, \quad \lambda_{0}=0.5 \mu \mathrm{m}, \quad n_{e}=2.3, \quad r_{33}=280 \mathrm{pm} / \mathrm{V} \text {. }
$$

Thus, in this paper, one $x$ or $y$ unit corresponds to $7.96 \mu \mathrm{m}$, one $z$ unit corresponds to $3.66 \mathrm{~mm}$, and one $E_{0}$ unit corresponds to $12.76 \mathrm{~V} / \mathrm{mm}$ in physical units. In the experiments, the applied voltage is $-550 \mathrm{~V} / 5 \mathrm{~mm}$, which gives $E_{0}=8.62$ in our numerical simulations.

It should also be noted here that in our experimental results the diffraction length can be approximately evaluated (for the beams widths and wavelengths that we typically used) as being $2.5 \mathrm{~mm}$. As our crystal extends over $10 \mathrm{~mm}$ in the z-direction, it is clear that the patterns that we observe are over a few (roughly 4) diffraction lengths and hence if they are self-supported within such length scales, this will indicate that they are indeed self-trapped beams. On the other hand, as we will see in what follows (in our numerical simulations), for all the configurations that we will find to be unstable, the instability development will arise typically for dimensionless propagation distances of $10<z<100$. Since these distances are considerably longer (in dimensional units) than the propagation distance in our crystal, all of the patterns presented in sections 35 below (even the most unstable ones) should, in principle, be experimentally observable in our setting. This is corroborated by our experimental results in section 6. 
The numerical simulations are done with a uniform spatial mesh with $\Delta x=$ $1 / 3$ and domain size $30 \times 30$, i.e. $91 \times 91$ grid points for most configurations (see Fig. 1 for a schematic of the spatial configurations). For some of the configurations, a larger domain was required, in which case a domain of size $60 \times 60$, i.e. $181 \times 181$ grid points, was used. Regarding the typical dynamics of a soliton when it is unstable, we simulate the z-dependent behavior using a Runge-Kutta fourth-order method using a step $\Delta z=0.00025$.

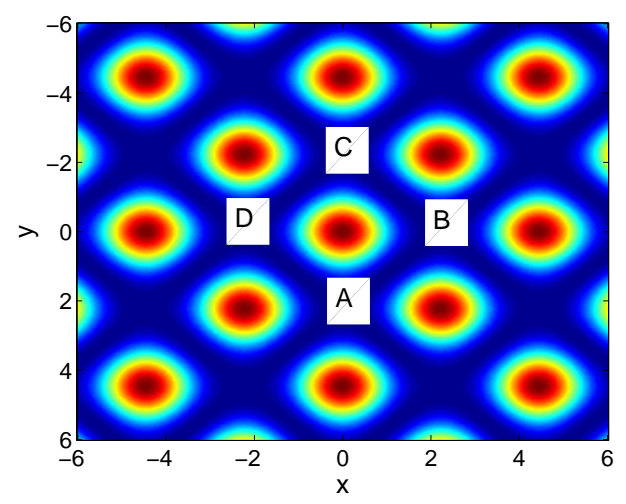

Fig. 1. (Color online) A spatial (x-y) contour plot of the ordinary polarization standing wave [lattice beam in Eq. (1)]. The localized pulses will be sitting at the minima of the lattice field, as opposed to the focusing nonlinearity lattice field, where they reside at the maxima. Points $A, B, C$, and $D$ are used for naming the dipole configurations. $A$ is a nearest-neighbor minimum of $B$ and $D$ (in our "diagonally-oriented" lattice), while it is a next-nearest-neighbor of $C$. Because of that, dipoles whose two lobes are at $A$ and $B$ (or at $C$ and $D$ ) will be called nearest-neighbor dipole solitons. The ones that are sitting at $A$ and $C$ (or at $B$ and $D)$ will be called next-nearest-neighbor dipoles.

Stationary solutions of Eq. (1) are sought in the form of $U(x, y, z)=u(x, y) e^{i \mu z}$, where $\mu$ is the propagation constant and $u$ is a real valued function satisfying

$$
\mu u-\left(u_{x x}+u_{y y}\right)-\frac{E_{0}}{1+I_{o l}+u^{2}} u=0 .
$$

The localized states $u(x, y)$ of (3) were obtained using the Newton-GMRES fixed point solver nsoli from [21] and continuation was used as a function of $\mu$, to follow the relevant branches of solutions.

The propagation constant $\mu$ we consider in this report is in the first spectral gap. Using Hill's method for the 2D problem [22], for parameter values mentioned above, we find the band gap to be $4.2 \lesssim \mu \lesssim 5.462$.

2 We note that the linear spectrum for $\mu$ quoted here and shown in the following images was the most accurate we computed using Hill's method based on consistency over $\Delta x$ in the limit $\Delta x \rightarrow 0$. The linear spectrum for the discretized problem with 
The power or the norm of the solitary waves is defined as follows:

$$
P=\left[\int_{-\infty}^{\infty} \int_{-\infty}^{\infty}|U|^{2} d x d y\right]^{1 / 2}
$$

We analyze the linear stability of soliton solutions $u(x, y)$ of (3) by applying an

infinitesimal perturbation to them. Writing $U(x, y, z)=e^{i \mu z}\left(u(x, y)+e^{\lambda z} \tilde{u}(x, y)\right)$, the perturbation $\tilde{u}(x, y)$ will then satisfy the following linearized equation

$$
i \lambda \tilde{u}=\mu \tilde{u}-\left(\tilde{u}_{x x}+\tilde{u}_{y y}\right)-\frac{E_{0}}{\left(1+I_{o l}+u^{2}\right)^{2}}\left[\left(1+I_{o l}\right) \tilde{u}-u^{2} \tilde{u}^{*}\right],
$$

where the superscript $*$ denotes complex conjugation. We solve the above linear eigenvalue problem using MATLAB's standard eigenvalue solver package.

At this point, it is also relevant to summarize the results of the discrete NLS defocusing model of [17] that we will use for comparison with the findings below. These results are incorporated in Table 1, where the stability of all the possible combinations of in-phase and out-of-phase, nearest-neighbor and next-nearest-neighbor configurations is quantified in terms of their relevant eigenvalues of linearization. The configurations are dubbed unstable when they possess (for all parameter values) real eigenvalue pairs, whereas they are considered marginally stable, when they do not always have such pairs. However, the latter configurations typically, in this setting, possess imaginary eigenvalues with negative Krein signature (see e.g. [23] and references therein), which practically means that if these collide with other eigenvalues, as $\mu$ is varied, complex eigenvalue quartets will emerge out of Hamiltonian-Hopf bifurcations [24], destabilizing the relevant solution. Hence, such solutions are not always linearly unstable, but may become unstable for some parameter ranges.

\section{Nearest-neighbor Dipole Solitons}

In this section, we report dipole solitons where the two lobes of the wave are located in two nearest-neighbor $(\mathrm{NN})$ lattice sites in the 2D square lattice potential shown in Fig. 1. The lobes can have the same phase or $\pi$ phase difference and are, accordingly, hereafter termed in-phase (IP) dipoles and

our chosen discretization is slightly different however, and in particular, the first band edge occurs at a slightly larger value of $\mu$. It is interesting to note that all saddle-node bifurcations occur at the accurate band edge, while those solutions which degenerate to linear Bloch modes actually degenerate beyond this value at the band edge particular to the discretization (not shown). 
Table 1

\begin{tabular}{|l|c|c|}
\hline & NN Stability & NNN Stability \\
\hline IP Dipole & Stable $\left(N_{i}^{-}=1\right)$ & Unstable $\left(N_{r}=1\right)$ \\
\hline OOP Dipole & Unstable $\left(N_{r}=1\right)$ & Stable $\left(N_{i}^{-}=1\right)$ \\
\hline IP Quadrupole & Stable $\left(N_{i}^{-}=3\right)$ & Unstable $\left(N_{r}=3\right)$ \\
\hline OOP Quadrupole & Unstable $\left(N_{r}=3\right)$ & Stable $\left(N_{i}^{-}=3\right)$ \\
\hline
\end{tabular}

Summary of the stability results of the discrete model with cubic nonlinearity, studied in [17], for all the in-phase (IP) and out-of-phase (OOP), nearest-neighbor (NN), as well as next-nearest-neighbor (NNN) configurations. $N_{r}$ denotes real eigenvalue pairs and $N_{i}^{-}$denotes imaginary eigenvalue pairs with negative Krein signature.

out-of-phase (OOP) dipoles, respectively. Notice that due to the (diagonal) nature of our lattice, the nearest-neighbor configurations that we consider are "built" along the diagonal direction.

\subsection{In-phase Nearest-neighbor Dipole Solitons}

We have found IP dipoles in a large interval of propagation constants $\mu$ for the given voltage $E_{0}$. We found that the solitons exist for $\mu$ smaller than 5.46, or for peak intensities larger than 0.144 . We note that the intensity of the dipoles cannot be arbitrary low, a result similar to the observed results of the focusing case $[5,20]$. The relevant findings are summarized in Fig. 2.

The top left panel of Fig. 2 shows the stability of the dipoles as a function of the propagation constant $\mu$, by illustrating the maximal growth rate (maximum real part over all perturbation eigenvalues) of perturbations. When $\max (\operatorname{Re}(\lambda))=0$, this implies stability of the configuration, while the configuration is unstable if $\max (\operatorname{Re}(\lambda)) \neq 0$ in this Hamiltonian system. We found that the stability region of this type of dipoles is given by $4.2 \leq \mu \leq 4.91$, the left hand limit corresponding to the Bloch band. The top right panel depicts the peak intensity and the power of the dipoles.

The middle left and right panels show the profile $u$ of a dipole at $\mu=5$ and the corresponding spectra at the complex plane, respectively. We see that the soliton is unstable due to an oscillatory instability. This is the typical instability in this case, in line with the discrete cubic model results. Clearly, there is an imaginary eigenvalue with negative Krein signature [23] which upon collisions with the continuous spectrum results in Hamiltonian-Hopf bifurcations and concomitant oscillatory instabilities.

As we increase $\mu$ further, the dipoles disappear in a saddle-node bifurcation. The bifurcation diagram is depicted in the top panels of Fig. 2. At the bifurca- 

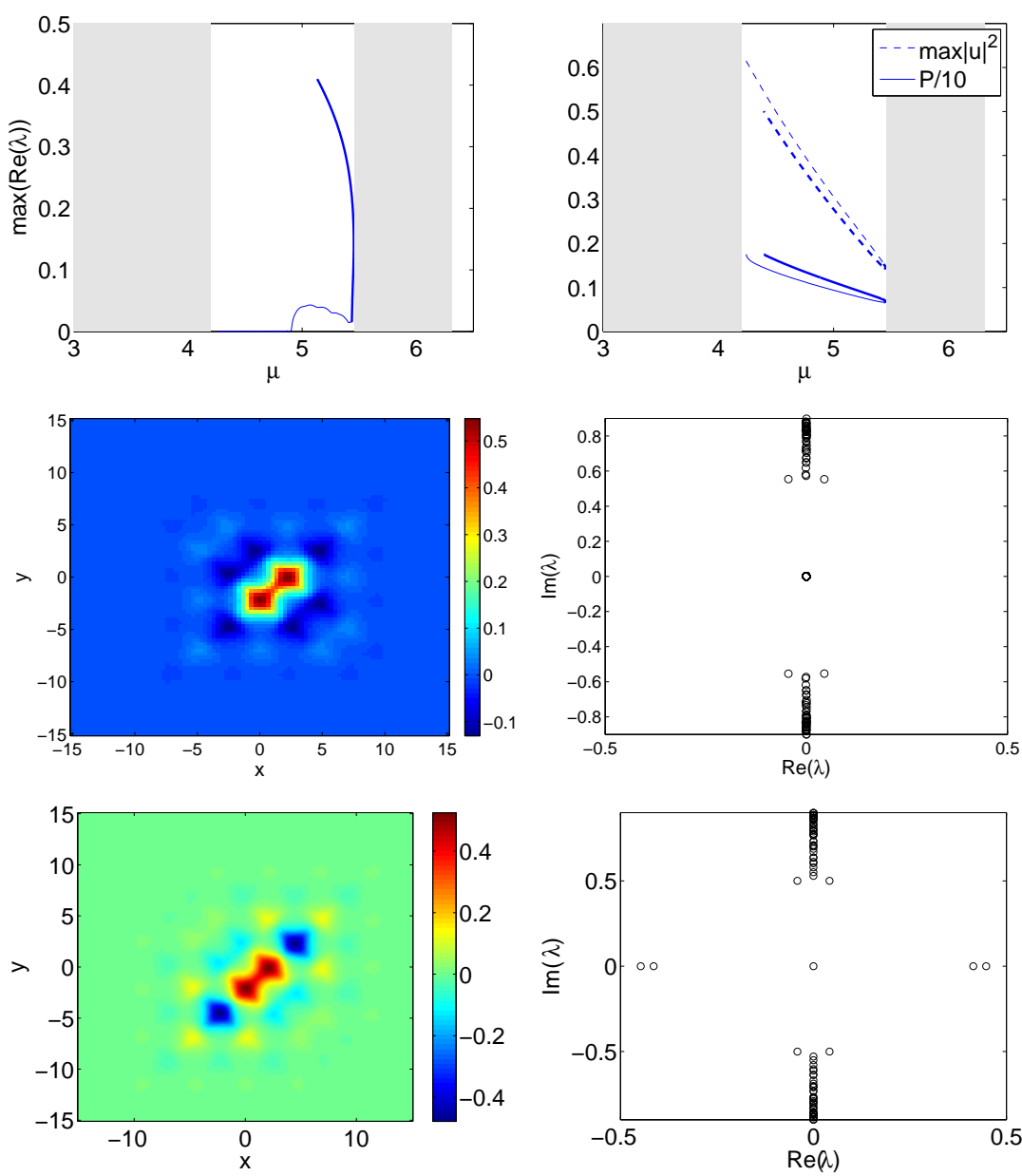

Fig. 2. (Color online) The top left panel shows the stability of the dipoles as a function of the propagation constant $\mu$. It is stable when the spectra is purely imaginary (i.e., when $\max (\operatorname{Re}(\lambda))=0$ ). The top right panel depicts the peak intensity and the power of the dipoles. The thin line corresponds to the solution represented in the middle row, while the bold line corresponds to the waveform illustrated in the bottom row. These two branches of solutions collide and mutually annihilate in a saddle-node bifurcation. The shaded areas in both of these panels represent the bands of the periodic potential. The middle (resp. bottom) left and right panels show the profile $u$ of the branch indicated by thin (resp. bold) line in the top row at $\mu=5$ and the corresponding complex spectral plane $(\operatorname{Re}(\lambda), \operatorname{Im}(\lambda))$ of the eigenvalues $\lambda=\operatorname{Re}(\lambda)+i \operatorname{Im}(\lambda)$. 

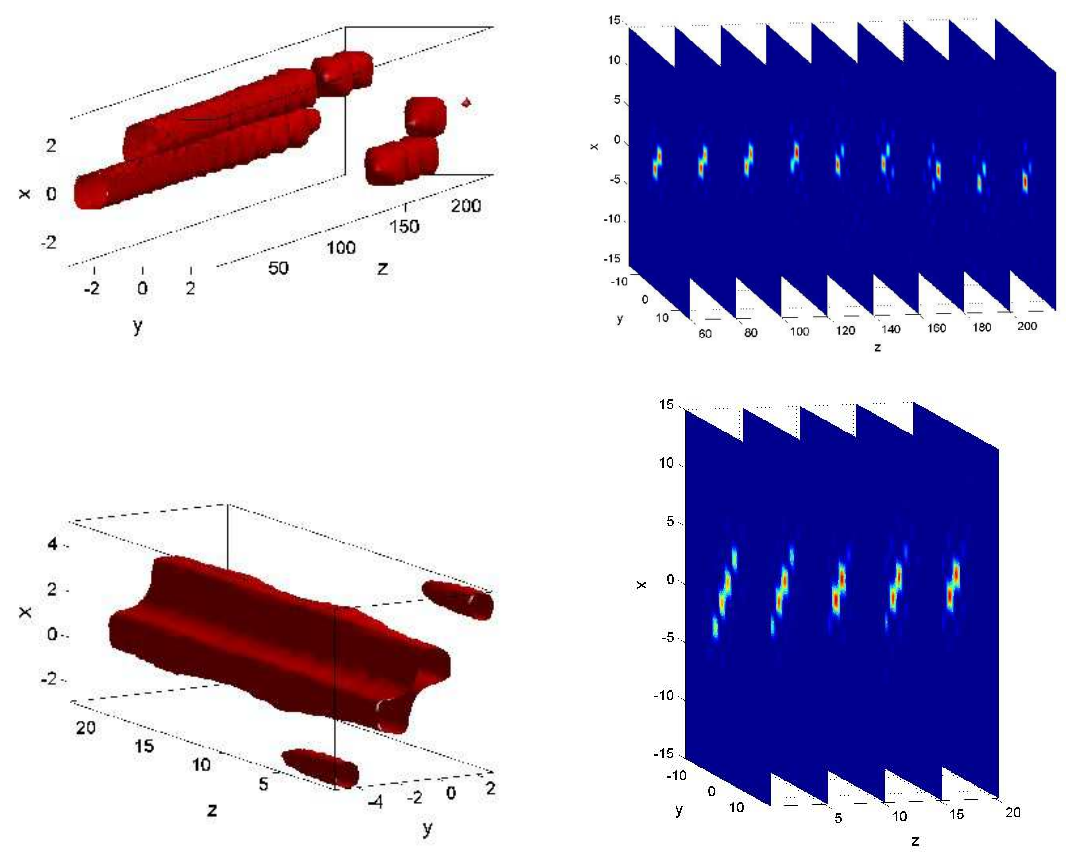

Fig. 3. (Color online) The evolution of the dipoles shown in Fig. 2 perturbed by a random noise of maximum intensity $0.25 \%$ of the soliton peak intensity. Presented in the figure are the isosurfaces (left panels) of height 0.2 for the first configuration (top panels) and of height 0.1 for the second one (bottom panels) and their slices at some instances (right panels).

tion point, $d P / d \mu \rightarrow \infty$, as $\mu \rightarrow 5.46$, i.e., at the boundary of the first Bloch band. At this point, the IP NN configuration collides with a configuration shown at the bottom panel of Fig. 2 (where the two nearest-neighbors -along the axis of the dipole- of the two populated wells become out-of-phase with them) and disappears in a saddle-node bifurcation. The corresponding profile and spectral plane for the saddle branch at $\mu=5$ is shown in the bottom right panel of the same figure, illustrating the strong instability of the latter.

We have also simulated the dynamics of the solitary waves when they are unstable. In Fig. 3 we present the evolution of the unstable dipoles shown in Fig. 2. The dipoles are perturbed by a random noise with maximum intensity $0.25 \%$ of the soliton peak intensity. Shown in Fig. 3 are the isosurfaces and the slices of the soliton along the propagation direction.

The dynamics of the soliton shown in the middle panel of Fig. 2 is presented in the top panels of Fig. 3. One can see that even with that strong perturbation, at $z=100$ the soliton still resembles its initial configuration. Physically, this corresponds to a propagation distance of approximately $366 \mathrm{~mm}$. This means that the instability is very unlikely to be observed in the photorefractive crystal lattice used in our experiments. For longer propagation distances, the oscillatory instability sets in and finally rearranges the dipole into a fun- 
damental soliton type configuration principally centered around a single site.

For the dipole shown in the bottom panel of Fig. 2, we present its dynamics as bottom panels of Fig. 3. We found that the instability is strong as predicted above such that even after a relatively short propagation distance, the configuration turns into the more stable solution of the IP NN dipole branch. We did not present the further dynamics of the dipole, as it is similar to the upper panels.

\subsection{Out-of-phase Nearest-neighbor Dipole Solitons}

We have also found OOP dipoles arranged in nearest-neighboring lattice wells. We summarize our findings in Fig. 4 where one can see that the solitons exist in the whole entire region of propagation constant $\mu$ in the first Bragg gap, $\mu \in(4.2,5.46)$. This smooth transition indicates that the OOP NN dipole solitons emerge out of the Bloch band waves; see e.g. [25] and [26] for a relevant discussion of the 1D and of the 2D problem respectively, in the case of the cubic nonlinearity. Nonetheless, the OOP NN dipoles are typically unstable due to a real eigenvalue pair. Notice that this is in agreement with the prediction of the discrete model (as can be seen from Table 1).

As the branch merges with the band edge, we observe an interesting feature, namely that the configuration resembles that of a quadrupole with a $\pi$ phase difference between two neighboring solitons, which we call +-+- quadrupoles below. This can be an indication that these structures bifurcate out of the Bloch band from the same bifurcation point. We elaborate this further in our discussion of the quadrupole structures in section 5 .

In Fig. 5 we present the instability dynamics of an OOP NN dipole soliton perturbed by similar random noise perturbation as in Fig. 3. This type of dipoles is typically more unstable than its IP counterpart, as is illustrated in the figure. In particular, in this example of unstable evolution even at $z=10$, the instability already manifests itself. One similarity of the instability of OOP NN dipoles with that of the IP NN ones is that the dipoles tend to degenerate to a single-site, fundamental gap soliton, which is stable in this setting. We note in passing that similar evolution results for dipoles and quadrupoles (but for short propagation distances) in the focusing case are discussed in [20]. 

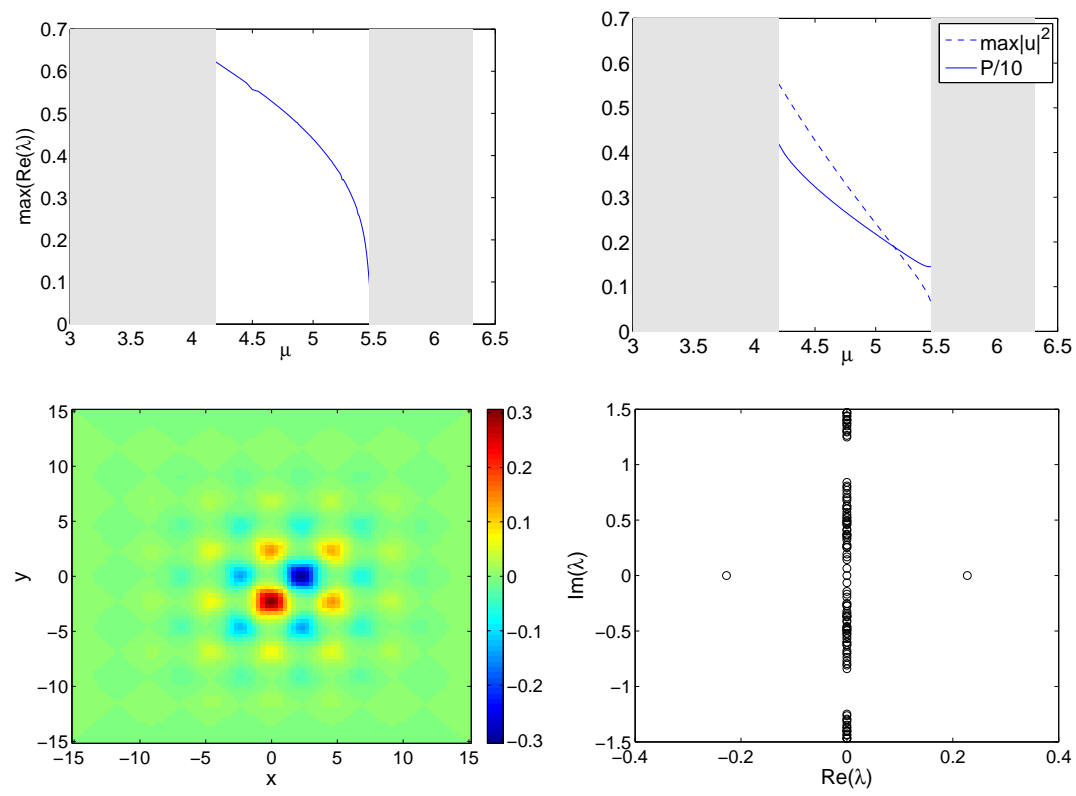

Fig. 4. (Color online) The top panels correspond to the same panels of Fig. 2 but for OOP NN dipole solitons. The bottom panel shows the profile $u$ and the corresponding spectra in the complex plane of the dipoles at $\mu=5.4$.
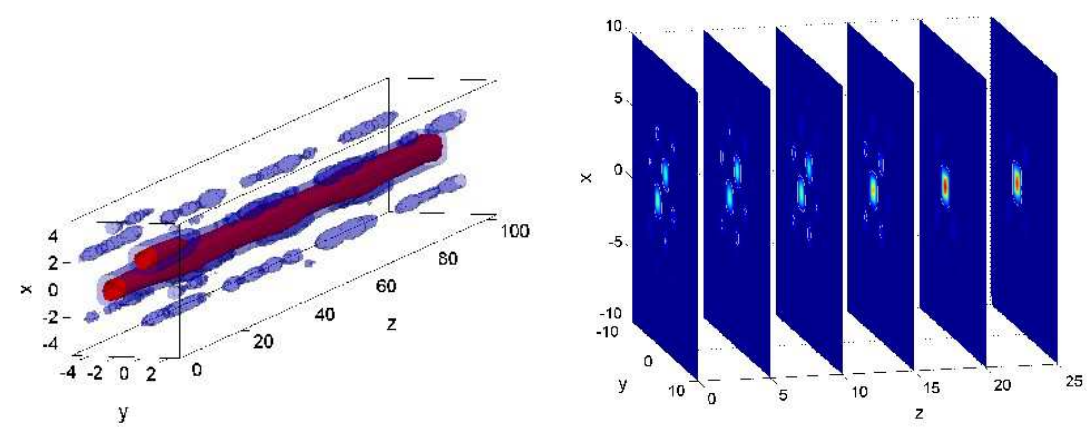

Fig. 5. (Color online) Similar to Fig. 3 but for the evolution of OOP NN dipoles. Shown are the isosurfaces of height 0.05 (red) and 0.015 (blue) and the contour plot slices at some select propagation distances.

\section{Next-nearest-neighbor Dipole Solitons}

We have also obtained dipole solutions that are not oriented along the two nearest-neighboring lattice wells, but rather where the two humps of the structure are located at two adjacent next-nearest-neighboring lattice sites. These humps can once again have the same phase or $\pi$ phase difference between them. We will again use the corresponding IP and OOP designations for these next-nearest-neighbor (NNN) waveforms. Notice here that NNN configurations that we consider are among the "closest" next-nearest-neighbor pairs, i.e., with the structures being aligned horizontally. In principle, one can con- 
sider more remote pairs of next-nearest-neighbors (e.g., along the diagonal), however the main qualitative stability properties discussed below would not change in such a case.

\subsection{In-phase Next-nearest-neighbor Dipole Solitons}
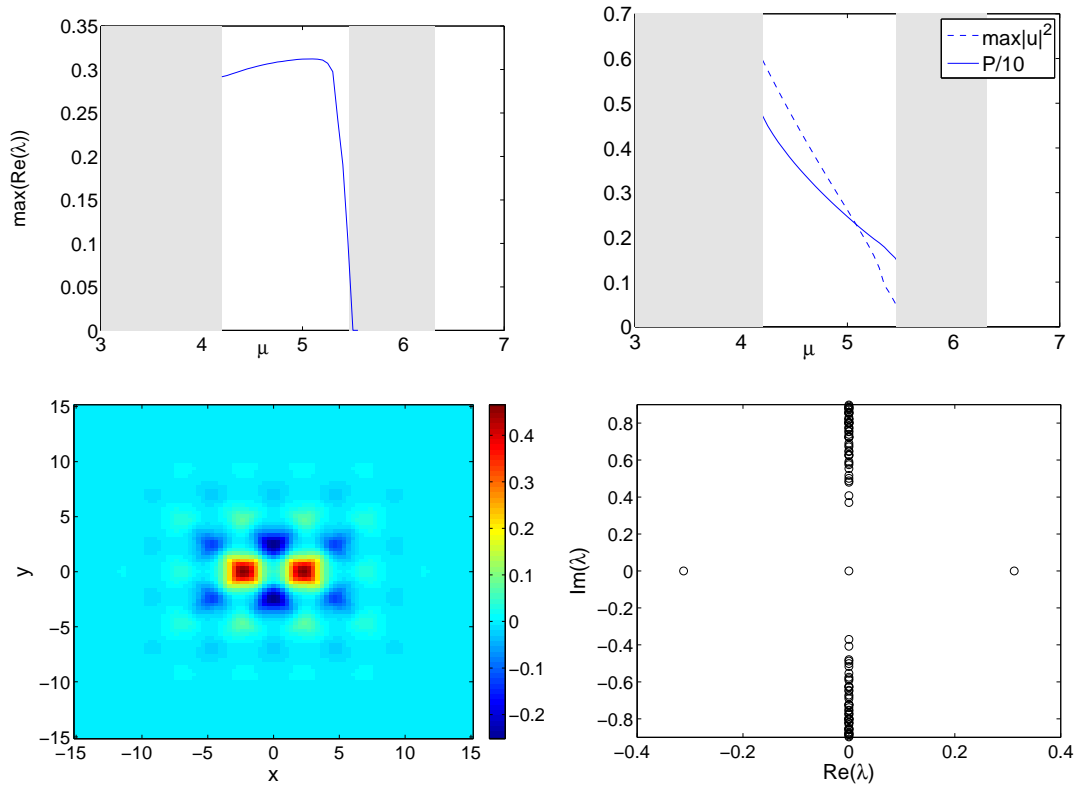

Fig. 6. (Color online) The top panels correspond to the same diagnostics as in Fig. 2 but for IP NNN dipole solitons. The bottom panels show the profile $u$ and the corresponding spectral plane of the IP NNN dipole at $\mu=5.1$.
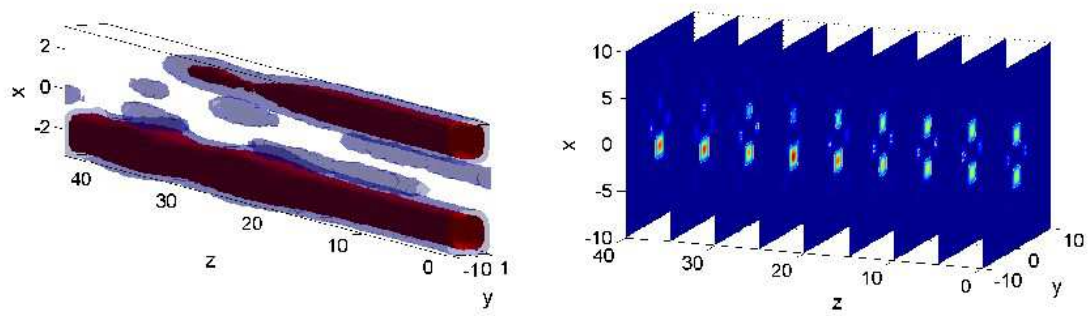

Fig. 7. (Color online) Similar to Fig. 3 but for the evolution of the IP dipole shown in Fig. 6. Presented are the isosurfaces of height 0.1 (red) and 0.05 (blue) and the sliced contours at some selected propagation distances.

We have obtained this type of IP, NNN dipole solitons in a wide parameter range. The stability, the power and peak intensity of these dipoles are shown in Fig. 6. These dipoles typically possess a real eigenvalue (again in line with the discrete cubic model prediction of Table 1). 
If one compares these IP NNN dipole solitons with the IP NN dipole solitons of Fig. 2, there are clear differences, such as the typical instability (with a real eigenvalue) of the former in contrast with the typical stability of the latter (possessing an imaginary eigenvalue of negative signature that can potentially become unstable upon collision with another eigenvalue). Another important difference is that the present IP dipoles emerge out of the Bloch band edges, contrary to what is the case for their IP NN counterparts where the solitons disappear through a saddle-node bifurcation. Interestingly, as the branch approaches the upper band-edge, the profile of an IP NNN dipole becomes similar to that of $\mathrm{a}+-+-$ quadrupole (see section 5.1 below) and of an OOP NN dipole, as the relevant limit is approached.

In Fig. 7, we present the dynamical evolution of the IP NNN dipole shown in Fig. 6 under similar random noise perturbation as above. Here, we also see that the instability appears earlier than the IP NN dipoles. The two humps deform until they become one hump already at the propagation distance $z \approx 20$, transforming the dipole solution into a single-hump gap soliton.

\subsection{Out-of-phase Next-nearest-neighbor Dipole Solitons}

We have also obtained OOP NNN dipole solitons. A typical profile of this family of solutions for $\mu=5.3$ is shown in Fig. 8. The power diagram of these solitons is presented in the top panel of Fig. 8. One important finding in this case is that there is a relatively wide stability region, i.e., typically these structures are stable (as indicated again by the comparison with the results of Table 1). We have found that the stability range for $E_{0}=8.62$ is $4.15 \lesssim \mu \lesssim 5.17$. This class of solutions typically suffers an oscillatory instability due to the presence of a single eigenvalue with negative signature and its collision with the continuous spectrum, as shown in the middle right panel of Fig. 8 (cf. once again with the discrete model prediction of Table 1).

For this solution also, we observe that similarly to the IP NN dipoles, it disappears at a non-zero peak intensity, in particular for $\max \left(|u|^{2}\right) \approx 0.11$. The disappearance is because of collision of this dipole with another configuration shown in the bottom panels of Fig. 8 in a saddle-node bifurcation. It is relevant to note that the point of the bifurcation is very close to the edge of the Bloch band, i.e., for $\mu \approx 5.46$.

Subsequently, we simulate the dynamics of the instability and present it in Fig. 9. One can see that for the OOP NNN, tunneling occurs during the propagation of the soliton along the $z$-direction, finally leading to localization at a well different from the original support of the dipolar structure. Regarding the counterpart solution of the other branch, the evolution along the $z$-direction is 

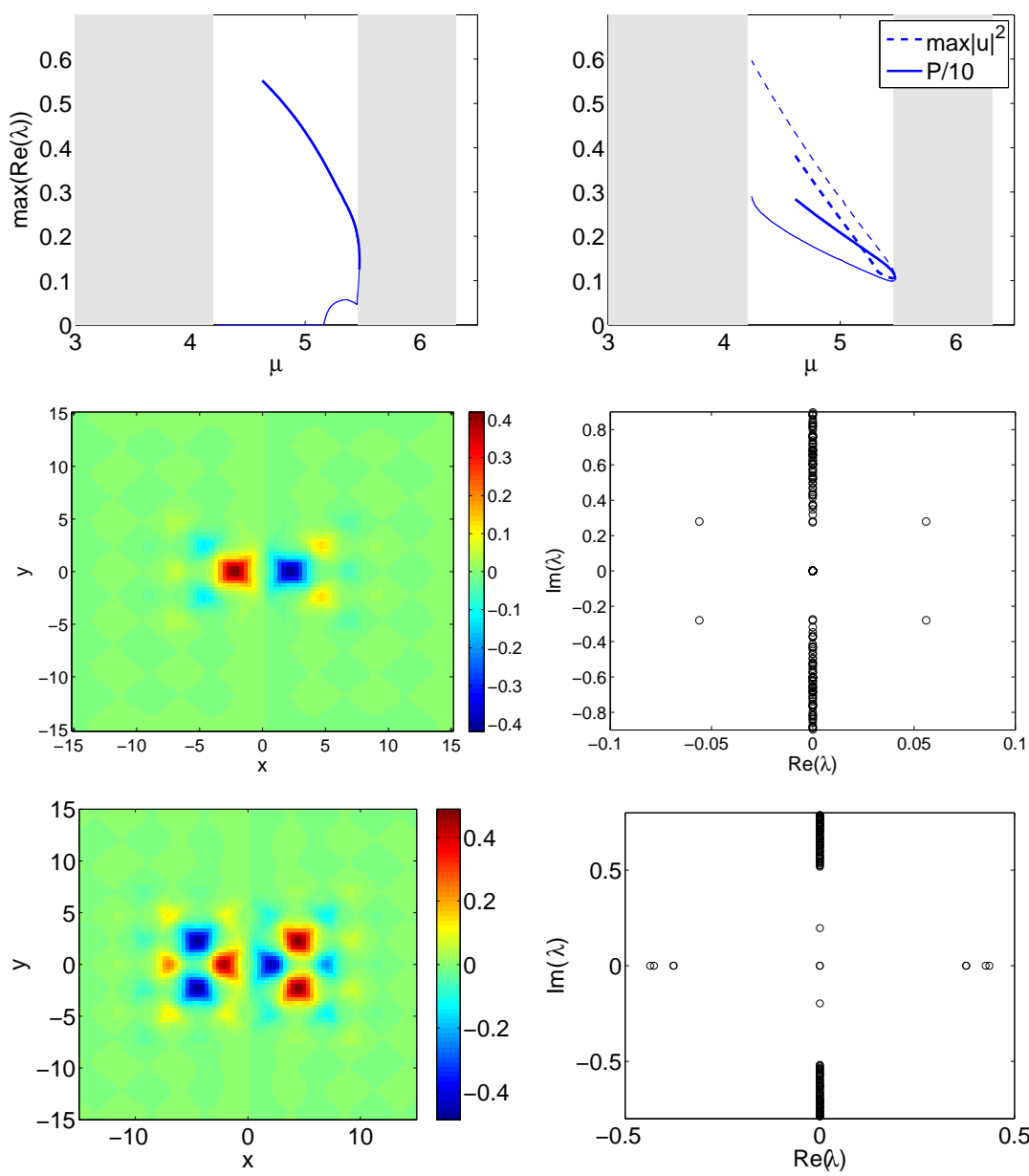

Fig. 8. (Color online) The top panels depict the largest real part of the critical eigenvalue, as well as the power and the peak intensity of the OOP NNN dipole solitons. The middle panels show the profile $u$ and the corresponding spectra in the complex plane of the dipole at $\mu=5.3$, and the bottom is the unstable saddle configuration, which collides with the OOP NNN profile in a saddle-node bifurcation, shown for $\mu=5$. 

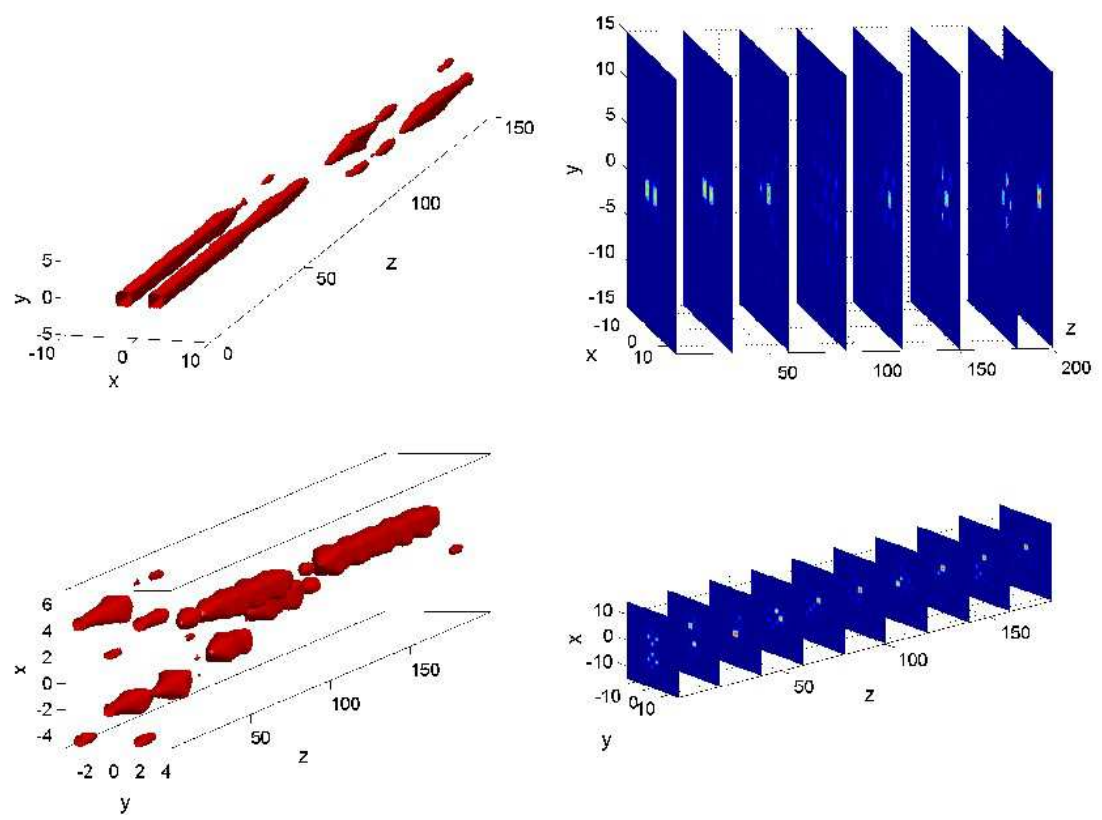

Fig. 9. (Color online) The evolutions of the OOP NNN dipole and its corresponding counterpart for the same propagation constant $\mu$, i.e. $\mu=5.3$, shown in Fig. 6 . Presented are the isosurface of height 0.1 and the slices at particular propagation distances where one can see clearly that the light tunnels away from its initial lattice wells.

a bit different as there is no tunneling away from the original position. Yet, this configuration also leads to localization at a single site in its final configuration.

\section{$5 \quad$ Nearest-neighbor Quadrupole Solitons}

We now turn to the examination of quadrupolar structures with configurations having four lobes at four adjacent lattice wells. Such structures turn out to exist in a large parameter region as well. In particular, we will focus our discussion on the case of such nearest-neighbor quadrupoles.

Before we proceed with the discussion of our findings, we will explain the notations that we use for the quadrupole configurations. Since there are four humps with the phase difference between two neighboring wells being either 0 or $\pi$, we have four possible configurations (up to rotational and phase symmetries), i.e.,,+-+-+--+++++ , and +--- , where ' + ' and '-' represent the sign of the excitation of the main hump of $u$, i.e. whether it is positive (phase 0) or negative (phase $\pi$ ), respectively, at the four excited sites A, B, C and D of Fig. 1. 


\section{$5.1+-+-$ Nearest-neighbor Quadrupole Solitons}

First, we consider the +-+- type. In Fig. 10, we present a field profile of this structure for $\mu=5$. We found that this configuration exists in the entire forbidden-band $\mu \in(4.2,5.46)$, i.e., OOP $\mathrm{NN}$ quadrupoles also bifurcate from the edge of the Floquet band. Nonetheless, we observe that a typical instability that these structures have is 3 real eigenvalue pairs (two of which are coincident in the bottom right panel of Fig. 10), in line with the predictions of Table 1.
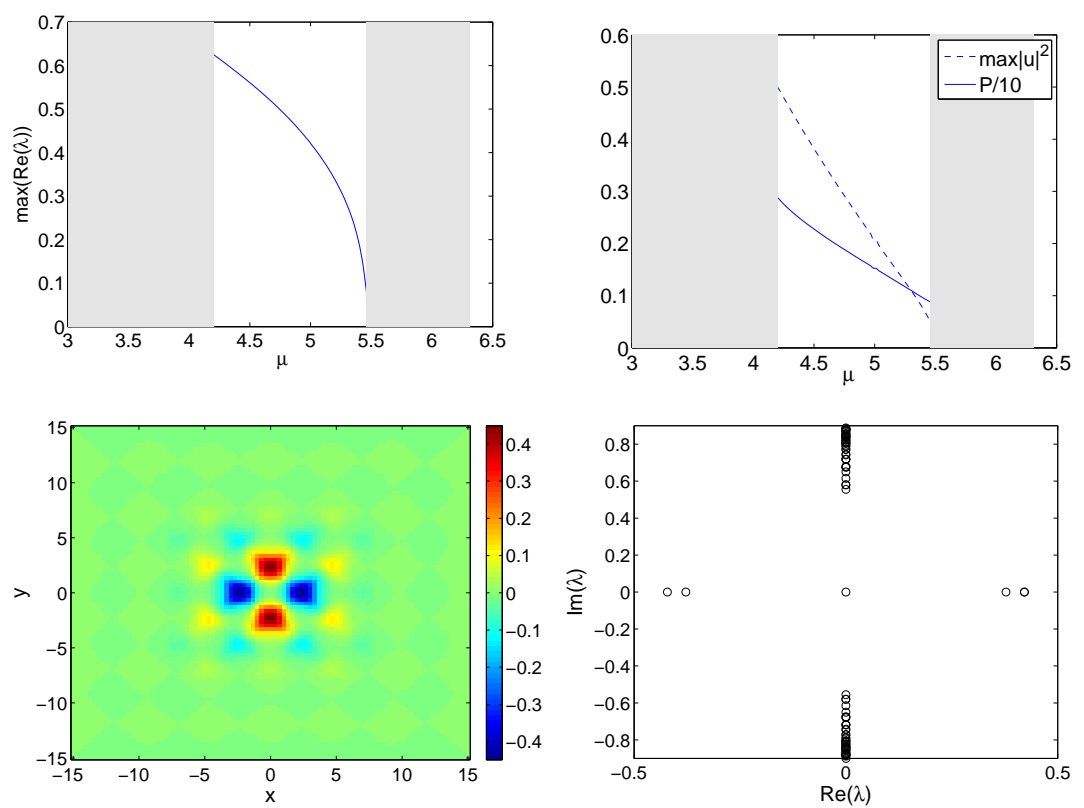

Fig. 10. (Color online) The maximum of the real part of the critical eigenvalue (top left panel) and the power and the peak intensity of the +-+- quadrupoles (top right panel). The bottom panels depict the profile $u$ of a $+-+-\mathrm{NN}$ quadrupole at $\mu=5$ and the corresponding linearization spectral plane of eigenvalues.

In Fig. 11, we present the evolution of the unstable +-+- quadrupole shown in Fig. 10. One can see that the configuration is strongly unstable resulting in a breakup of the structure already for $z \approx 10$ to a IP NNN dipole state. Subsequent evolution also visits the other state associated with the quadrupole branch, namely the OOP NN and eventually results into a single hump gap soliton.

\section{2 + + ++ Nearest-neighbor Quadrupole Solitons}

++++ quadrupoles have four in-phase humps at adjacent lattice sites. A typical example of this sort is shown in Fig. 12 at $\mu=5$. We have analyzed the stability of this configuration, finding that it is stable in a wide parametric range, namely for $4.09 \lesssim \mu \lesssim 4.93$, in line with what is predicted by the cubic 

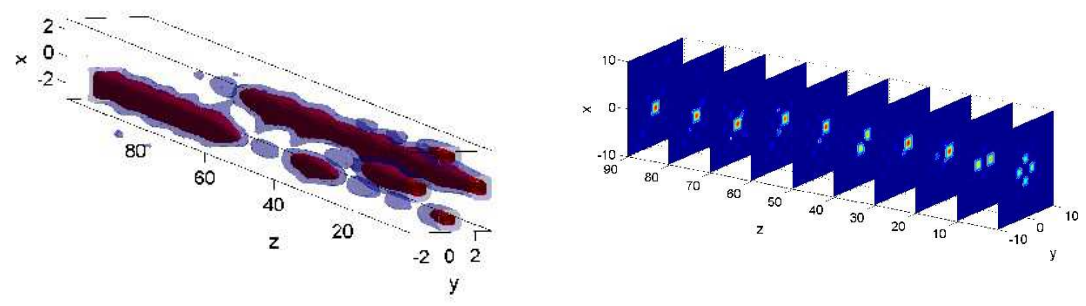

Fig. 11. (Color online) The evolution of the quadrupole presented in Fig. 10. Shown are the isosurfaces of height 0.15 (red) and 0.07 (blue) and its slices at some selected propagation distances.

nonlinearity discrete model in Table 1 . Similarly to the IP NN and the OOP NNN branch this branch disappears at a non-zero peak intensity (in this case, for $\left.\max \left(|u|^{2}\right) \approx 0.16\right)$.
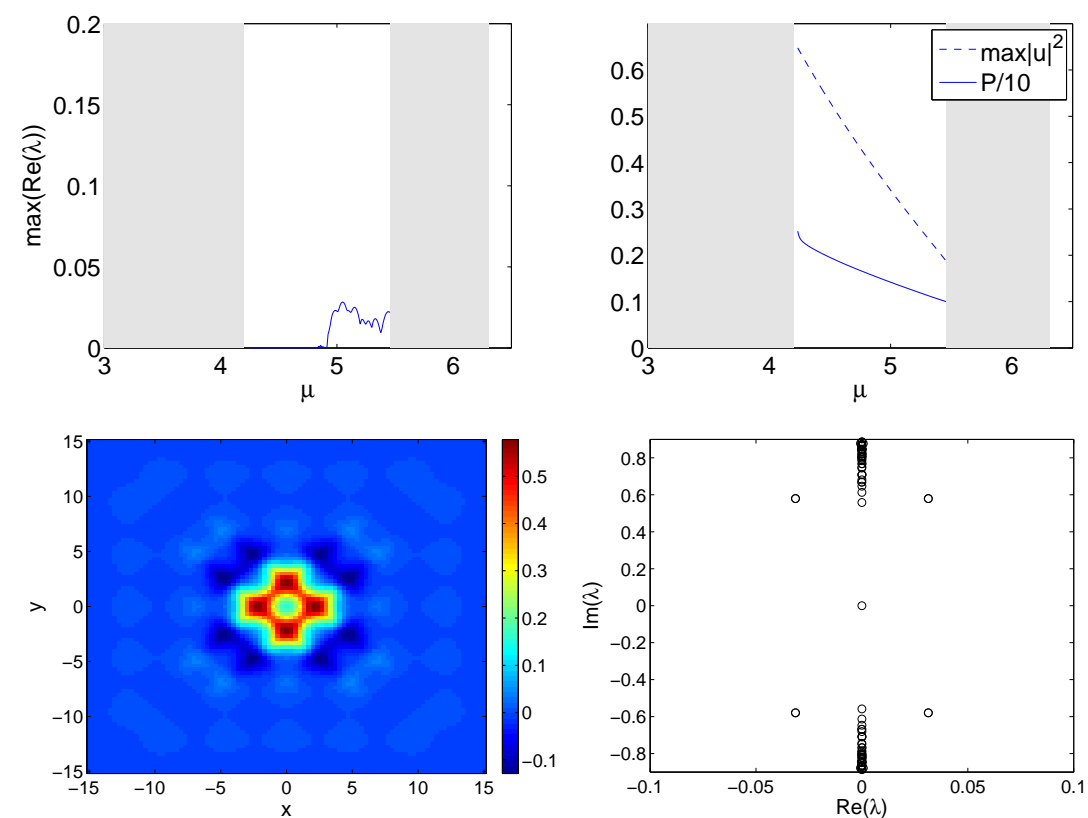

Fig. 12. (Color online) The same as Fig. 10 but for the ++++ quadrupoles. The top panels show the maximal instability growth rate of perturbations (left panel) and the branch optical power and maximal intensity (right panel), while the bottom panels show the solution profile (left) and linear stability (right panel) for $\mu=5$.

The dynamics of the configuration when it is unstable has also been simulated; as a particular example, we integrate the field profile shown in Fig. 12 in $z$. Even though it is unstable, we found that propagation even for 100 units of (dimensionless) length, the profile still resembles its initial condition. This seems to indicate that such structures should be rather straightforward to observe experimentally. For sufficiently long propagation, the dipole will eventually be destroyed through an oscillatory instability. 

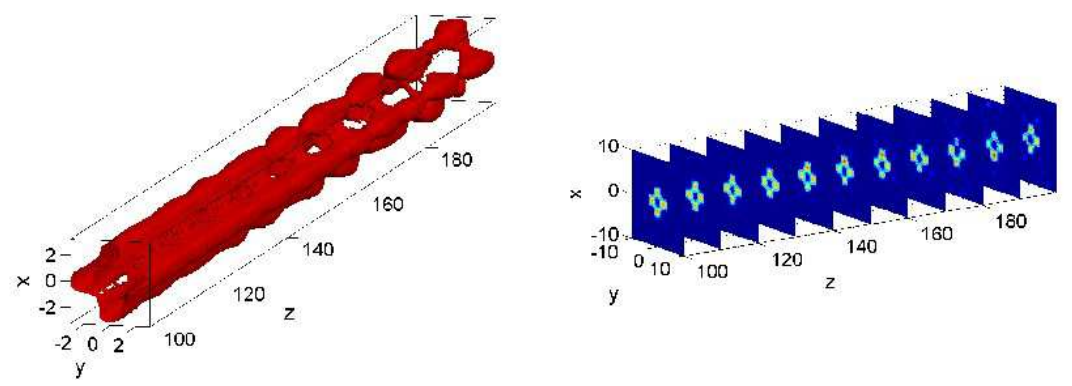

Fig. 13. (Color online) The evolution of the quadrupole presented in Fig. 12. Shown are the isosurfaces of height 0.2 (red) and 0.15 (blue) and its slices at particular propagation distances.

\section{$5.3+--+$ and +- - Nearest-neighbor Quadrupole Solitons}

One can also examine other types of quadrupole configurations such as +--+ or +++- . However, one then typically finds, as may be inferred by their nonsymmetric profile, that these configurations are either always unstable (as is e.g., the case with +--+ ) or are not straightforward to obtain numerically in a steady state form (as is e.g., the case with +--- ). As a representative example of these asymmetric profiles, we show in the middle panels of Fig. 14 the case of the +--+ profile, which is always unstable due to two real eigenvalue pairs (and potentially also oscillatorily unstable).

Note that this configuration is equivalent to the superposition of two IP NN (or two OOP NN, etc.) dipoles. Another saddle node bifurcation happens in this case as the solution approaches the band edge, leading to the collision of this branch with a more extended saddle configuration shown in the bottom panels of Fig. 14.

In this case also, observing the dynamics of the instability in Fig. 15, we have seen the relatively fast degeneration of the mode into a single site solitary wave profile. The corresponding evolution dynamics for quadrupoles in the focusing case (but for short propagation distances) can be found in [20].

\section{Experimental Results}

In our experiments, we use a setup similar to that used in [27]. A partially spatially incoherent beam (of wavelength $488 \mathrm{~nm}$ ) is produced by use of a rotating diffuser. A negatively biased photorefractive crystal (SBN:60 6x10x5 $\mathrm{mm}^{3}$ ) provides a self-defocusing nonlinearity. An amplitude mask is also used to spatially modulate the otherwise uniform beam after the diffuser, in order to produce a $2 \mathrm{D}$ periodic lattice. The mask is then imaged onto the input face 

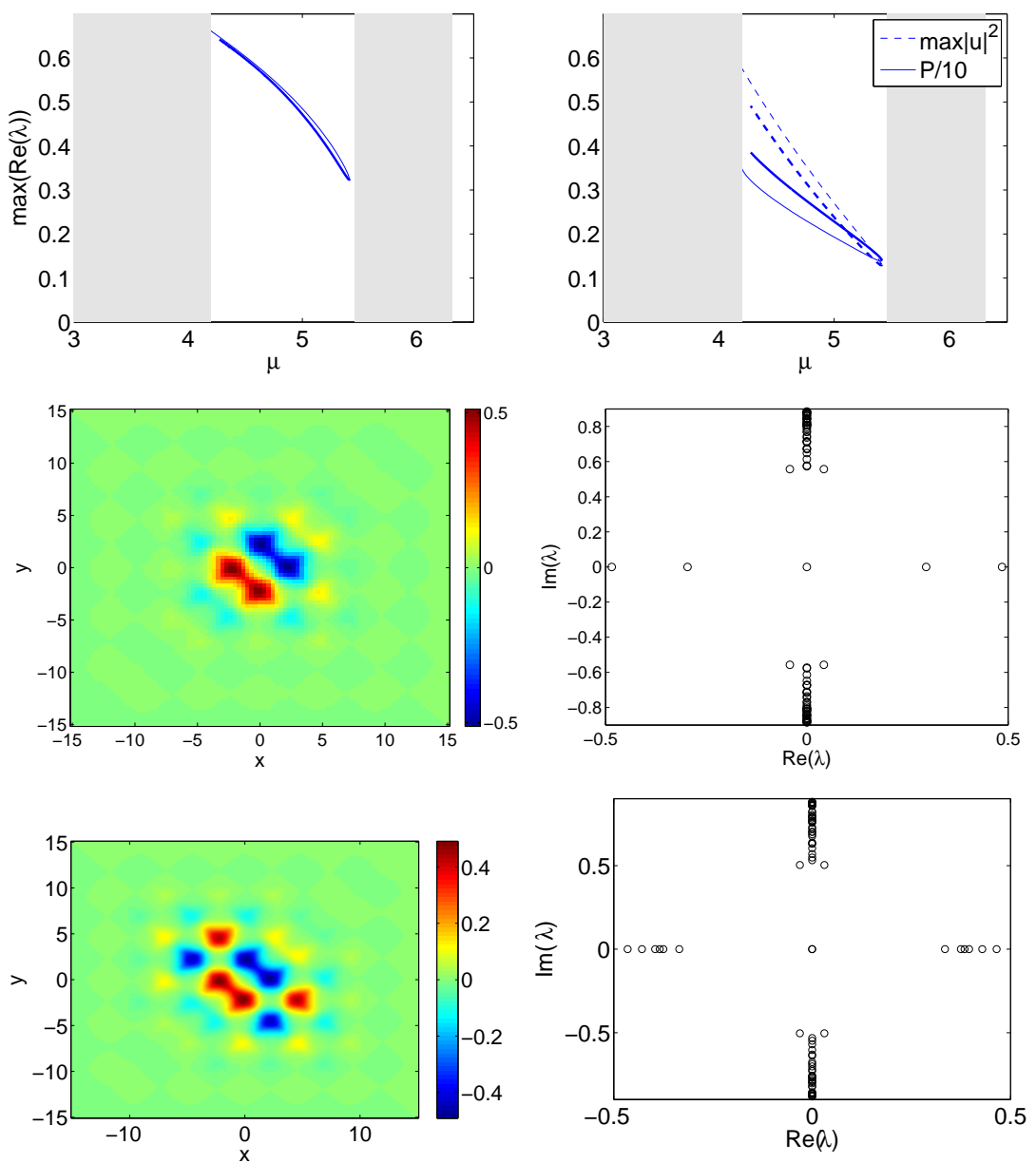

Fig. 14. (Color online) The top four images are the same as Fig. 10 but for the +--+ quadrupoles; once again the thin lines of the top row correspond to the solution profile and spectral plane of the middle row, while the bold lines of the top row to the solution profile and spectral plane indicated in the bottom row.

of the crystal, thus creating a pixel-like input intensity pattern with a spatial period of about $25 \mu \mathrm{m}$. The ensuing lattice beam (represented by $I_{o l}$ in our theoretical model) is diagonally oriented and ordinarily polarized, thus the induced waveguide arrays remain invariant during propagation. An extraordinarily polarized beam splitting from the same laser output is used as the probe beam (this is the complex field $u$ that we monitor in our analysis above). The probe beam (intensity about 6 times weaker than that of the lattice beam) is sent into a Mach-Zehnder interferometer to create a dipole-like or quadrupolelike input pattern whose phase is controlled with the piezo-transducer (PZT) mirrors. The input/output intensity patterns and the k-space power spectra of the lattice and soliton-forming beams are monitored with CCD cameras.

By employing a defocusing nonlinearity, the two-dimensional pixel-like intensity pattern induces a backbone waveguide lattice whose intensity minima correspond to the waveguide sites [2,28]. Launching two narrow Gaussian beams 

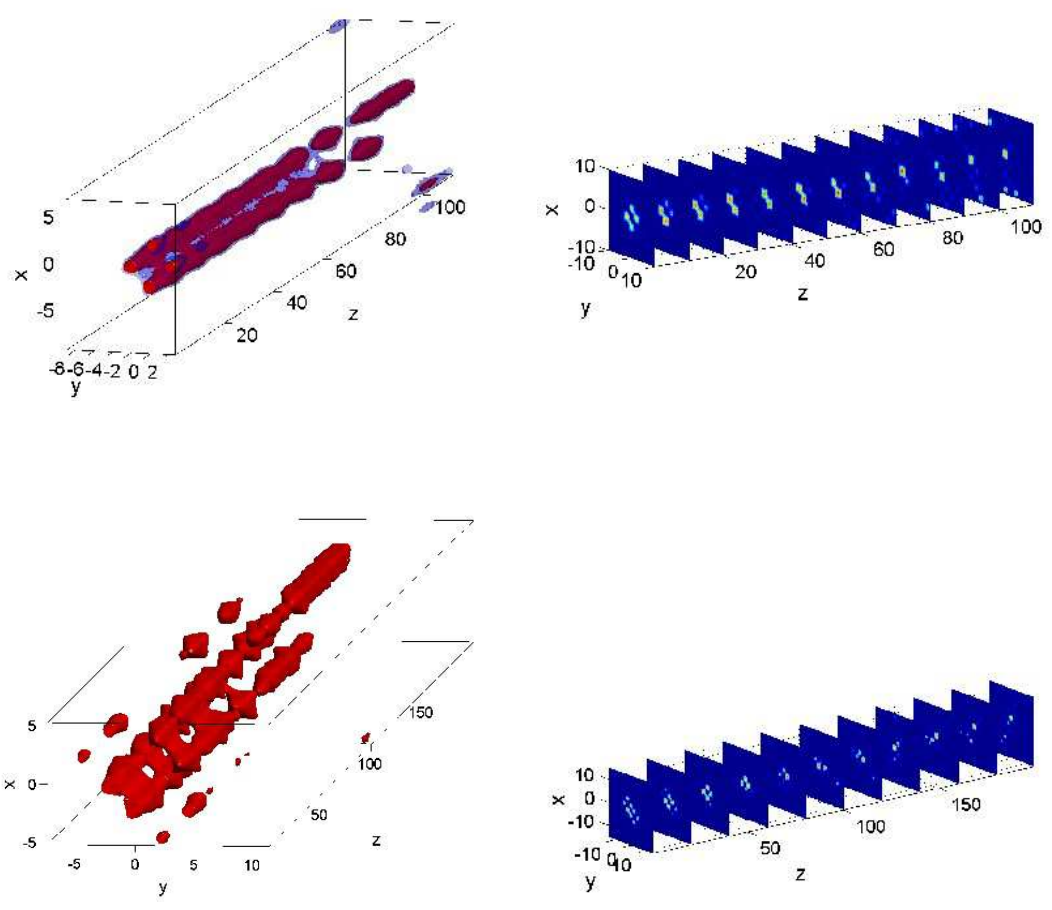

Fig. 15. (Color online) The evolution of the quadrupoles presented in Fig. 14. Top panels show the isosurfaces of height 0.2 (red) and 0.15 (blue) and the contour at select propagation distances of the configuration in the middle panels of Fig. 14 . Bottom panels are the corresponding figures for the counterpart soliton. The isosurface shown is of height 0.1 . Both configurations eventually give rise to single-site localization.

into two nearest-neighbor or next-nearest-neighbor waveguide sites either with in-phase or with out-of-phase relations, the probe beams evolve into dipolelike gap solitons through the $10 \mathrm{~mm}$ SBN crystal under a proper strength of nonlinearity. Typical results are shown in Fig. 16, where the output intensity patterns, upon propagation through the crystal (first column), show two main bright spots. This indicates that the energy of the probe beams is mostly localized in the waveguides that were initially excited. The interferograms between these output patterns and a tilted plane wave show that these two main spots remain in-phase or out-of-phase, maintaining their initial phase relation, although the secondary intensity peaks adjacent to the main ones are always out-of-phase with the primary spots. The spatial spectra (Fourier transform) of the patterns (third column) are in good agreement with those obtained from the Fourier transform of the corresponding theoretical solutions (last column). It is important to note here that although some of these configurations such as the OOP NN and the IP NNN dipoles (second and third rows in Fig. 16) have been found to always be unstable, the limited propagation distances inside the crystal $(10 \mathrm{~mm}$ which corresponds to $z \approx 2.73$ in our dimensionless units) are too short for the instability to develop to an observable degree in 
the experiment, while it is clearly shown in the theoretical analysis of soliton solutions in the previous sections.

We have also performed experiments to excite quadrupole-like gap solitons, with four narrow Gaussian beams launched into four adjacent waveguide sites (i.e., four adjacent intensity minima) for both in-phase and out-of-phase conditions. Under a proper level of defocusing nonlinearity, self-trapping is observed with four principal intensity peaks localized in the waveguides initially excited [Fig. 17(a)] for both in-phase (top) and out-of-phase (bottom) conditions. Furthermore, the measured spatial spectra [of Fig. 17(b)] for these quadrupole-like gap solitons are also in good agreement with the corresponding ones obtained from the theoretical solutions [as shown in Fig. 17(c)].

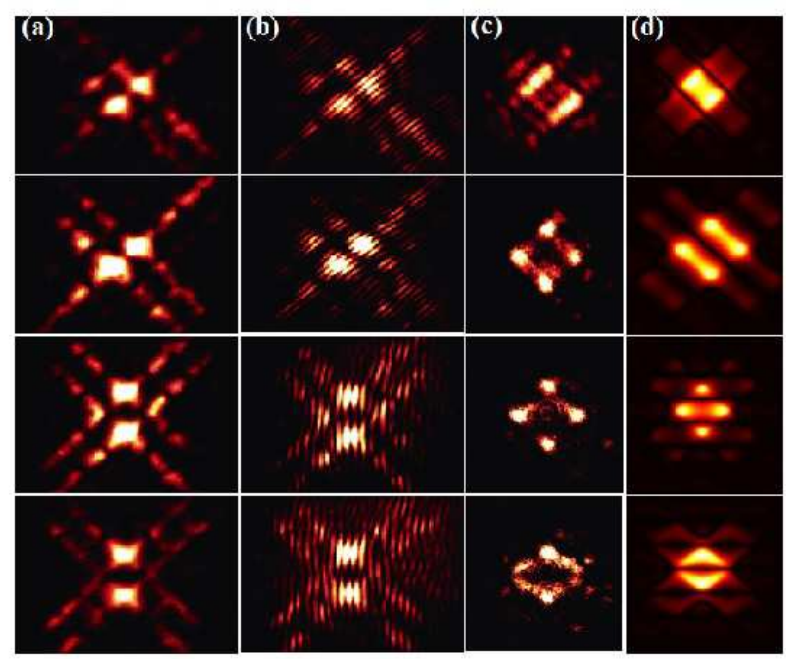

Fig. 16. (Color online) Observations of dipole like gap soliton. From top to bottom: IP NN, OOP NN, IP NNN, and OOP NNN. Panel (a) shows the output intensity patterns, (b) shows the interferagrams of these patterns with a tilted plane wave, (c) shows the spatial (Fourier) spectra and, (d) shows those spectra from theoretical calculations of the corresponding solutions.

\section{Conclusions}

In this communication, we examined in detail theoretically and numerically the existence, stability and dynamics of multipole lattice solitons excited with a saturable defocusing photorefractive nonlinearity. In this connection, we have obtained a wide array of relevant structures, including dipoles and quadrupoles, examining both the different possible configurations (in-phase, as well as out phase profiles), as well as cases where the excited sites are at nearest or at next-nearest-neighboring lattices sites. 


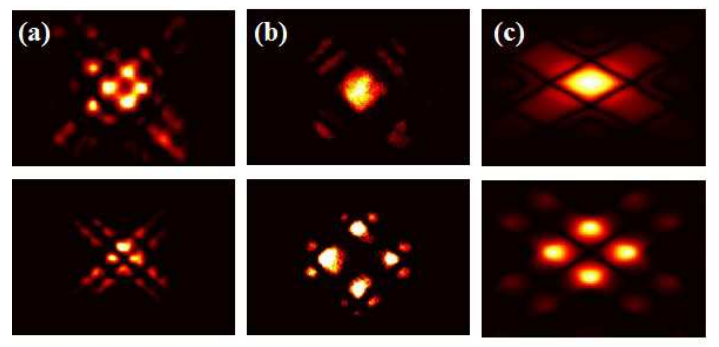

Fig. 17. (Color online) Observations of in-phase (top) and out-of-phase (bottom) quadrupole-like gap solitons. Panel (a) shows the output intensity patterns, (b) shows the spatial spectra, and (c) shows those spectra from theoretical calculations of the corresponding profiles.

We have found configurations such as the in-phase, nearest-neighbor dipole, the out-of-phase, next-nearest-neighbor dipole or the in-phase nearest-neighbor quadrupole which are typically stable (although they may incur oscillatory instabilities). We have also identified those solitons including e.g., the in-phase, next-nearest-neighbor dipole, the out-of-phase, nearest-neighbor dipole or the out-of-phase nearest-neighbor quadrupole which are typically unstable due to exponential instabilities and real eigenvalues. The stability results have been found to be in direct agreement with the results of the analysis of the prototypical discrete model of this type, namely the discrete nonlinear Schrödinger equation. Furthermore, we have also identified an interesting set of bifurcations that are associated with the parametric continuation and termination of some of the above branches. The dynamical instabilities encountered in the present work have been monitored through direct integration of the relevant dynamical equation. In most cases, the result of evolution has been the degeneration of the structure to a robust single site solitary wave, although more complicated oscillatory evolutions are also possible.

Our experimental results agree with the theoretical predictions. Different dipole and quadrupole configurations were realized in the experiment in a photorefractive crystal with limited propagation length, even the most unstable ones. This is simply because the growth rate of the dynamical instabilities is such that the instabilities will manifest themselves typically for propagation distances on the order of $100-1000 \mathrm{~mm}$, but they are not appreciable within the short distance comparable to the length of our crystal.

Since the framework of defocusing equations has been studied far less exten- 
sively than their focusing counterparts, it would be particularly interesting to extend the present considerations to other structures. Perhaps the most interesting example would be the study of discrete vortices, not only of single but also of multiple charge which would be an interesting endeavor both from a theoretical, as well as from an experimental point of view. Such structures could be directly connected to ones studied theoretically also in other fields, including e.g. weakly interacting Bose-Einstein condensates; relevant examples of vortices of charges 1 and 2 are given by the tightly bound structures of Figs. 8 and 10 respectively of [29]. Additionally, the present considerations of defocusing nonlinearity may be extended to other settings, including the case of radial lattices of [8]. In the latter case, one would expect to observe modulationally stable, radial beam waveforms with or without vorticity [30,31]. Such studies are currently in progress and will be reported in future publications.

Acknowledgements. This work was supported by the 973 Program, NSFC and PCSIRT in China, and by US NSF and AFOSR (ZC). We thank J. Yang for discussions. PGK gratefully acknowledges support from NSF-DMS-0505663, NSF-DMS-0619492 and NSF-CAREER.

\section{References}

[1] N.K. Efremidis, S. Sears, D. N. Christodoulides, J. W. Fleischer, and M. Segev Phys. Rev. E 66, 46602 (2002).

[2] J.W. Fleischer, M. Segev, N.K. Efremidis and D.N. Christodoulides, Nature 422, 147 (2003); J.W. Fleischer, T. Carmon, M. Segev, N.K. Efremidis and D.N. Christodoulides, Phys. Rev. Lett. 90, 23902 (2003).

[3] D. Neshev, E. Ostrovskaya, Yu.S. Kivshar and W. Krolikowski, Opt. Lett. 28, 710 (2003).

[4] H. Martin, E.D. Eugenieva, Z. Chen and D.N. Christodoulides, Phys. Rev. Lett. 92, 123902 (2004).

[5] J. Yang, I. Makasyuk, A. Bezryadina, and Z. Chen, Opt. Lett. 29, 1662 (2004).

[6] J. Yang, I. Makasyuk, P. G. Kevrekidis, H. Martin, B. A. Malomed, D. J. Frantzeskakis, and Zhigang Chen, Phys. Rev. Lett. 94, 113902 (2005).

[7] D. Neshev, Yu. S. Kivshar, H. Martin, and Z. Chen, Opt. Lett. 29, 486-488 (2004).

[8] X. Wang, Z. Chen, and P. G. Kevrekidis, Phys. Rev. Lett. 96, 083904 (2006).

[9] D. N. Neshev, T.J. Alexander, E.A. Ostrovskaya, Yu.S. Kivshar, H. Martin, I. Makasyuk and Z. Chen, Phys. Rev. Lett. 92, 123903 (2004); J. W. Fleischer, G. 
Bartal, O. Cohen, O. Manela, M. Segev, J. Hudock, and D.N. Christodoulides Phys. Rev. Lett. 92, 123904 (2004).

[10] B. Freedman, G. Bartal, M. Segev, R. Lifshitz, D.N. Christodoulides and J.W. Fleischer, Nature 440, 1166 (2006).

[11] T. Schwartz, G. Bartal, S. Fishman and M. Segev, Nature 446, 52 (2007).

[12] D. N. Christodoulides, F. Lederer, and Y. Silberberg, Nature 424, 817 (2003); A. A. Sukhorukov, Y. S. Kivshar, H. S. Eisenberg, and Y. Silberberg, IEEE J. Quant. Elect. 39, 31 (2003).

[13] S. Aubry, Physica 103D, 201 (1997); S. Flach and C. R. Willis, Phys. Rep. 295, 181 (1998); D. K. Campbell, S. Flach, and Y. S. Kivshar, Phys. Today, January 2004, p. 43.

[14] V. A. Brazhnyi and V. V. Konotop, Mod. Phys. Lett. B 18, 627 (2004); P. G. Kevrekidis and D. J. Frantzeskakis, Mod. Phys. Lett. B 18, 173 (2004).

[15] O. Morsch and M. Oberthaler, Rev. Mod. Phys. 78, 179 (2006).

[16] B.B. Baizakov, B.A. Malomed and M. Salerno, Europhys. Lett. 63, 642 (2003); B.B. Baizakov, B.A. Malomed and M. Salerno, Phys. Rev. A 70, 053613 (2004); H. Sakaguchi and B.A. Malomed, Europhys. Lett. 72, 698 (2005).

[17] P.G. Kevrekidis, H. Susanto and Z. Chen, Phys. Rev. E 74, 066606 (2006).

[18] P.G. Kevrekidis, K.Ø. Rasmussen and A.R. Bishop, Int. J. Mod. Phys. B 15, 2833 (2001).

[19] J. Yang, New J. Phys. 6, 47 (2004).

[20] J. Yang, A. Bezryadina, I. Makasyuk and Z. Chen, Stud. Appl. Math. 113, 389 (2004).

[21] C. T. Kelley, Solving Nonlinear Equations with Newton's Method, no. 1 in Fundamentals of Algorithms, SIAM, Philadelphia, 2003.

[22] B. Deconinck and J. Nathan Kutz, J. Comp. Physics 219, 296-321, 2006

[23] T. Kapitula, P.G. Kevrekidis, B. Sandstede, Physica D 195263 (2004).

[24] J.-C. van der Meer, Nonlinearity 3, 1041 (1990).

[25] D. Pelinovsky, A.A. Sukhorukov, and Yu.S. Kivshar, Phys. Rev. E 70, 036618 (2004).

[26] Z. Shi and J. Yang, Phys. Rev. E 75, 056602 (2007).

[27] Z. Chen and K. McCarthy, Opt. Lett. 27, 2019 (2002).

[28] C. Lou, X. Wang, J. Xu, Z. Chen and J. Yang, Phys. Rev. Lett. 98, 213903 (2007).

[29] H. Sakaguchi and B.A. Malomed, J. Phys. B: At. Mol. Opt. Phys. 37, 2225 (2004). 
[30] Y.V. Kartashov, V.A. Vysloukh and L. Torner, Phys. Rev. Lett. 94, 043902 (2005).

[31] Q.E. Hoq, P.G. Kevrekidis, D.J. Frantzeskakis and B.A. Malomed, Phys. Lett. A 341, 145 (2005). 